\title{
Petri Net Modelling for Achieving an Optimal Design of a Wind Turbine Condition Monitoring System
}

\author{
Yan R.*, Dunnett S.J.** and Jackson L.M.*** \\ * Department of Aeronautical and Automotive Engineering, Loughborough University. Loughborough, Leicestershire \\ LE113TU,U.K; r.yan@lboro.ac.uk \\ ** Department of Aeronautical and Automotive Engineering, Loughborough University. Loughborough, Leicestershire \\ LE11 3TU, U.K; s.j.dunnett@lboro.ac.uk \\ *** Department of Aeronautical and Automotive Engineering, Loughborough University. Loughborough, \\ LeicestershireLE113TU,U.K; l.m.jackson@lboro.ac.uk
}

\begin{abstract}
Condition monitoring has demonstrated its effectiveness in improving the economic return of wind turbines. However, a wind turbine consists of hundreds, even thousands, of mechanical, electrical and power electronic components. The failure of any one of them may lead to the shutdown of the turbine. For this reason, a variety of component monitoring systems have been developed dedicated to monitoring these different components. Consequently, a wind turbine usually needs to be monitored simultaneously by several different types of component monitoring systems that benefit wind turbine operation and maintenance to different extents. This not only increases the complexity of the hardware configuration but also increases the costs of the entire condition monitoring system. How to achieve a condition monitoring system that can monitor the most vulnerable components whilst bringing the most economic benefit to the wind turbine operator is an important question. The aim of this paper is to answer such a question with the aid of the Petri net modelling method. The model developed in the paper will investigate the influences of condition monitoring systems and fault detection using wind farm Supervisory Control and Data Acquisition (SCADA) system on the economic return of wind turbines, thereby providing a feasible tool for constructing an optimal wind turbine condition monitoring system.
\end{abstract}

\section{Introduction}

Climate change has motivated the rapid development of renewable energy industries in the 21 st century. In April 2021, the European Union announced it will cut carbon emissions by at least 55\% by 2030, compared with 1990 levels (BBC, 2021). It is predicted that wind power will continue to play a vital role in achieving this challenging goal. Although most wind turbines are still onshore, an increasing number of wind turbines are being deployed offshore due to better wind resources, less noise pollution and land use issues at offshore sites (GWEC, 2019). Offshore wind farms have largely expanded during the past decade and it is expected to grow at a rate of more than $94 \mathrm{GW}$ per year until 2025 (GWEC, 2021). However, the current operation and maintenance (O\&M) costs of offshore wind turbines (OWTs) remains as high as $30 \%$ of the overall cost of energy (May et al., 2015). In particular, due to the remote locations of OWTs, their inspection and maintenance are much more difficult than for onshore ones.

In order to improve the reliability and availability of OWTs, condition monitoring techniques have been installed in many. Condition-Monitoring Systems (CMS) aim at detecting abnormal changes in the conditions that indicate a developing fault. The maintenance engineers are then able to perform the preventive maintenance based on the fault detected correspondingly, thereby avoiding catastrophic failures. This is known as condition-based maintenance (CBM). Many studies have been conducted to evaluate the feasibility and effectiveness of different Wind Turbine Condition-Monitoring Systems (WTCMS) for wind turbines. For example, Yang et al. proposed a new WT blade CM method based on the concept of transmissibility of frequency response functions (2015). Siegel et al. compared different vibration-based condition monitoring algorithms for wind turbine drive trains and discussed the effectiveness of these algorithms on the gear tooth problems and bearing-related failures, respectively (2014). Yang and Jiang investigated the potential values of Supervisory Control and Data Acquisition (SCADA) data for wind turbine condition monitoring and O\&M (Yang \& Jiang, 2011).

Despite these efforts, a fundamental question remains unanswered, i.e. how to achieve a condition monitoring system that can monitor the most vulnerable components whilst bringing the most economic benefit to the wind turbine operator. This paper aims to develop a mathematic simulation model using Petri 
nets (PN), which is able to evaluate the impact of different monitoring systems on the reliability, availability, and O\&M cost of the OWTs.

The remaining part of the paper is organised as follows. In Section 2, a typical OWT structure, the monitoring systems considered, and the maintenance strategies adopted are defined; In Section 3, the PN modelling technique is briefly reviewed; In Section 4, four different PN models are developed for describing the maintenance activities in offshore wind farms; In Section 5, the simulation results obtained using the PN simulation including the average number of repairs conducted in different scenarios and the corresponding maintenance costs are discussed; In Section 6, the paper concludes with key research findings and potential future works.

\section{Structure, Maintenance Strategies, and CMSs of the Offshore Wind Turbine}

\subsection{Structure of the OWT}

To facilitate mathematical modelling, a gear-driven OWT composed of six critical subsystems is considered in the research. The six subsystems are the rotor system, yaw and pitch (YP) system, drivetrain system, braking system, power system, and turbine structures, respectively. The brief description of each subsystem is given below.

- $\quad$ The rotor system is composed of the blades and the hub.

- The function of the yaw and pitch system is to adjust the blade pitch angle and orientation of the rotor to optimise the power output and maximise the wind energy conversion.

- $\quad$ The drivetrain system consists of the main shaft and a gearbox.

- The braking system locks the wind turbine position in non-operational mode during the maintenance of the wind turbine or extreme weather conditions. The braking system is used to slow down the rotor when the wind speed is beyond the cut-out speed of $25 \mathrm{~m} / \mathrm{s}$ (Dalgic et al., 2015; Abdollahzadeh et al., 2016).

- $\quad$ The power system converts mechanical torque into electrical power and transfer the frequency and voltage of the electricity generated to match grid requirements.

- $\quad$ The turbine structures include a nacelle, tower, and foundation.

In the study, it was assumed that any subsystem failure would cause the turbine to shut down. In addition, the health state of these subsystems is classified into three categories, i.e. healthy, fault, and failure. The wind turbine is able to operate normally if all the subsystems are in a healthy state. When a minor fault occurs in a subsystem, it is assumed that the subsystem is still able to work but it will generate abnormal conditions which could be detected by CMS. When a fault develops into a failure, the wind turbine will be shut down immediately. The natural degradation process of each subsystem is assumed to follow a Weibull distribution, and the parameters are derived based on the wind turbine failure rate data published in open literature (Le \& Andrews, 2016; Leigh \& Dunnett, 2016). The scale parameters ( $\eta$ ) in the distributions for computing the times from 'normal' to 'fault' and 'failure' are estimated based on the assumption that the time spans in the two scenarios will cover $70 \%$ and $30 \%$ of the Mean Time to Failure (MTTF) respectively of the corresponding subsystems. The MTTF of each subsystem is the inverse of the failure rate listed in Table 1.

\begin{tabular}{c|c}
\hline Subsystem & Annual failure rate (/year) \\
\hline Rotor & 0.0868 \\
\hline Drivetrain & 0.0600 \\
\hline Power system & 0.1430 \\
\hline YP system & 0.1534 \\
\hline Braking system & 0.0799 \\
\hline Structure & 0.0790 \\
\hline
\end{tabular}

TABLE 1. Failure rate of wind turbine subsystem 


\subsection{Maintenance strategy}

Three different maintenance strategies (Nakagawa, 2005), i.e. corrective maintenance, periodic maintenance, and condition-based maintenance, are adopted to improve the reliability and availability of the turbine. The corrective maintenance is conducted after a subsystem failure occurs. It should be noted that after corrective maintenance has been carried out, a full inspection of the turbine will be carried out. The condition-based maintenance is the maintenance carried out after a fault is detected. Hence, its effectiveness is highly dependent on the fault detection capability of the CMS installed in the wind turbine. To use maintenance resources (e.g. maintenance staffs, vessels, service time, etc.) more efficiently and minimise unnecessary investigations, the periodic maintenance in the study is delivered via two levels of service, namely 'basic service' (BS) and 'advanced service' (AS), as described in (Yan \& Dunnett, 2021). BS aims to fix the issues that may occur frequently but are difficult to monitor and may accelerate the degradation of the turbine components, such as the looseness of bolts, insufficient lubrication, etc. By contrast, AS requires more expensive maintenance resources to inspect all the subsystems of the wind turbine so that those faults that are not detected by the CMS can be revealed.

\subsection{Condition monitoring systems considered}

There are mainly two types of WTCMSs available in the commercial wind market (Yang et al., 2014). The first one is designed based on the Supervisory Control and Data Acquisition (SCADA) systems. The SCADA system integrated in all modern wind turbines is originally designed for the operation of wind turbines by monitoring energy generated and confirming the operation status of the turbine (Kuseyri, 2015). It records data using high frequency sampling and transmits 10 minutes averaged values back to the operators. The parameters monitored usually includes active power output, generator currents and voltages, wind speed, turbine and generator shaft speeds, gearbox bearing temperatures, generator bearing temperatures, average nacelle temperature and so on. Abnormal statuses in any of these components' conditions are able to trigger alarms. Many wind power companies have applied the SCADA-based condition monitoring because it is available at no or very low additional cost (Yang et al., 2014). However, these alarms could be too frequent if the detection margins are set to be too narrow. This is because the environmental conditions such as wind speed around the wind turbines are never constant and could change dramatically in a short period of time (especially at sea). In addition, SCADA systems do not collect all the signals which are capable to fully monitor the health statuses of all the turbine components. Also, data change such as an increase in the generator bearing temperature is a late stage indication of a fault, which means there might not be a large enough time window for repair before it develops to a failure.

The second one is the CMSs specifically designed for wind turbines. There have been many technologies, for example vibration analysis, oil particle counter, and ultrasonic testing, applied to the condition monitoring of wind turbines, some of which are already in commercial applications (Yang et al., 2014). Different CMSs with various capabilities are able to monitor different components/subsystems of the turbines. Although they do have a positive impact on ensuring the availability and reliability of wind turbines, their high cost is always an issue. In this paper, the CMS of the OWT considered consists of vibration analysis and oil particle counter whose cost is relatively low compared with others (García Márquez et al., 2012; Yang et al., 2014).

Both monitoring systems are installed in the OWT considered in the research. The subsystems they can monitor and their detection capabilities assumed are listed in Table 2 and are assumed based on expert knowledge and past literature (Yang et al., 2014). The detection capabilities include two aspects, i.e. (1) the time that the condition statuses monitored by the monitoring systems become abnormal; (2) the probabilities of successfully detecting the abnormal conditions. It should be noted that the time is assumed to follow a Weibull distribution and the scale parameters $\eta$ are estimated based on the percentage of the MTTF after the occurrence of the fault of each subsystem. 


\begin{tabular}{c|c|c|c|c}
\hline \multirow{2}{*}{ Subsystem } & \multicolumn{2}{|c|}{ SCADA system } & \multicolumn{2}{c}{ CMS } \\
\cline { 2 - 5 } & $\begin{array}{c}\text { MTTF } \\
\text { percentage after } \\
\text { fault }\end{array}$ & Detectability & $\begin{array}{c}\text { MTTF } \\
\text { percentage after } \\
\text { fault }\end{array}$ & Detectability \\
\hline Rotor & $20 \%$ & 0.50 & - & - \\
Drivetrain & $25 \%$ & 0.95 & 0 & 0.90 \\
Power system & $25 \%$ & 0.95 & 0 & 0.90 \\
YP system & $10 \%$ & 0.95 & 0 & 0.90 \\
Braking system & $10 \%$ & 0.60 & 0 & 0.90 \\
Structures & - & - & 0 & 0.90 \\
\hline
\end{tabular}

TABLE 2. Fault detection capability for each subsystem of the OWT

\section{A brief review of Petri net-based modelling technology}

To understand the PN models developed in this paper, the basics of the PN modelling method are briefly reviewed. A PN is a direct bipartite graph that consists of three types of symbols, namely circles, rectangles, and arrows as illustrated in Figure 1. Bigger circles represent the places, which are conditions or states of a system, such as failure. Coloured patterns inside the circles are used in order to differentiate between different places as described in (Yan \& Dunnett, 2021). The condition place, marked with yellow-horizontallines in the figure, means that the model will perform predefined actions if the conditions set for the place are met. The place filled by red-vertical-lines means that the simulation will be ended if a token is placed in it. Small solid black circles are used to represent tokens in the places. Rectangles represent the transitions, which are actions or events causing the change of condition or state. If the time of the transition is zero, the rectangle will be filled black, otherwise it is empty. Arrows, known as arcs, in the figure are used to connect places and transitions. Arcs with a slash on and a number, $n$, next to the slash represent a combination of $n$ single arcs and the arc is said to have a weight $\mathrm{n}$. The dashed arrow shown in the figure is a conditional arc. It means if the transition connecting to the arc is enabled, the probability of the expected tokens being produced in the output places is predefined. In addition, an arc with a small circle on one end is known as an inhibitor arc. This can prevent a transition from firing when enabled. A transition is enabled if the number of tokens in every input place is greater than or equal to the corresponding weights of the arcs to the transition. Once a transition is enabled, it will fire after the time associated with it and the tokens will be removed from the input places and put into the output places according to the weight of the corresponding arcs. The movement of the tokens gives the dynamic property of the PNs.

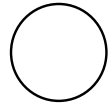

Normal place

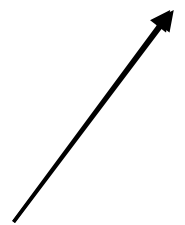

Normal arc

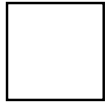

Timed transition

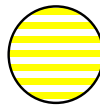

Condition place

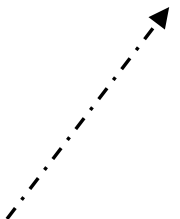

Conditional arc

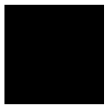

Instant transition

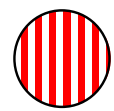

Terminate place

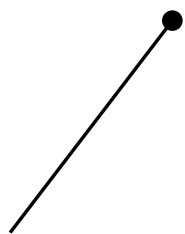

Inhibitor are

Token

FIGURE 1. Different symbols used in the PN models (Yan \& Dunnett, 2021) 


\section{Dynamic PN modelling of the O\&M of an offshore wind turbine}

The following four PN models are developed to simulate the O\&M of an OWT.

- Operation Petri net (OPN) - for simulating the normal operation and periodic maintenance of an OWT. In the OPN, the design life of the turbine and the interval of the periodic maintenance will be defined.

- $\quad$ System Petri net (SPN) - for simulating the degradation, the health state of the turbine subsystems over time, and the shutdown of the turbine due to failure.

- $\quad$ Detection Petri net (DPN) - for simulating fault detection by the monitoring systems.

- $\quad$ Recovery and Maintenance Petri net (RMPN) - for simulating the process to prepare and conduct the maintenance when a subsystem fails or a subsystem fault is detected.

These PNs work together and communicate with each other as required. It should be noted that the OPN and SPN are two core models for the simulation, their structures will not change over the process. The other two PN models (i.e. DPN and RMPN) will join the simulation only when they are required. This is achieved by importing new places, transitions, tokens, and connections (i.e. arcs) defined in DPN and RMPN models into the existing PN structure. They will be removed from the simulation when the assigned PN simulation task is completed or cancelled. The details of the nets are discussed in the following sections.

\subsection{Operational Petri Net (OPN)}

The OPN adapted from (Yan \& Dunnett, 2021) aims to simulate the normal operation and periodic maintenance of an OWT as shown in Figure 2. In the figure, the top part of the PN governs the normal operation of the wind turbine within the design life. Transition ' $\mathrm{S} 1$ ' represents the time the wind turbine is designed to operate, which is set to be 20 years because it is the typical design life of modern wind turbines (Tchakoua et al., 2014; Santos et al., 2015; Leigh \& Dunnett, 2016). A token produced in the place 'End of design life' after the firing of Transition ' $\mathrm{S} 1$ ' means the end of the operation of the wind turbine, which also refers to the end of one simulation.

The second part of the PN labelled 'periodic maintenance' is developed to simulate the periodic maintenance of the wind turbine. Both levels of services, i.e. AS and BS described in the previous sections, are included in the model. Transitions 'PM1' and 'PM3' are the time intervals of the two levels of services BS and AS respectively. In the study, they are set to be 6 months and 2 years respectively. Transitions 'PM2' and 'PM5' are the times required for performing the 'basic service' and 'advanced service', which are assumed to be 1 day and 2 days, respectively. It should be noted that the turbine will be stopped during the services. If a token is produced in the place, 'AS completed', the RMPN will be embedded into the model for preparing and conducting the maintenance if any fault was detected during the periodic maintenance. It is assumed that all the faults can be detected successfully in an AS.

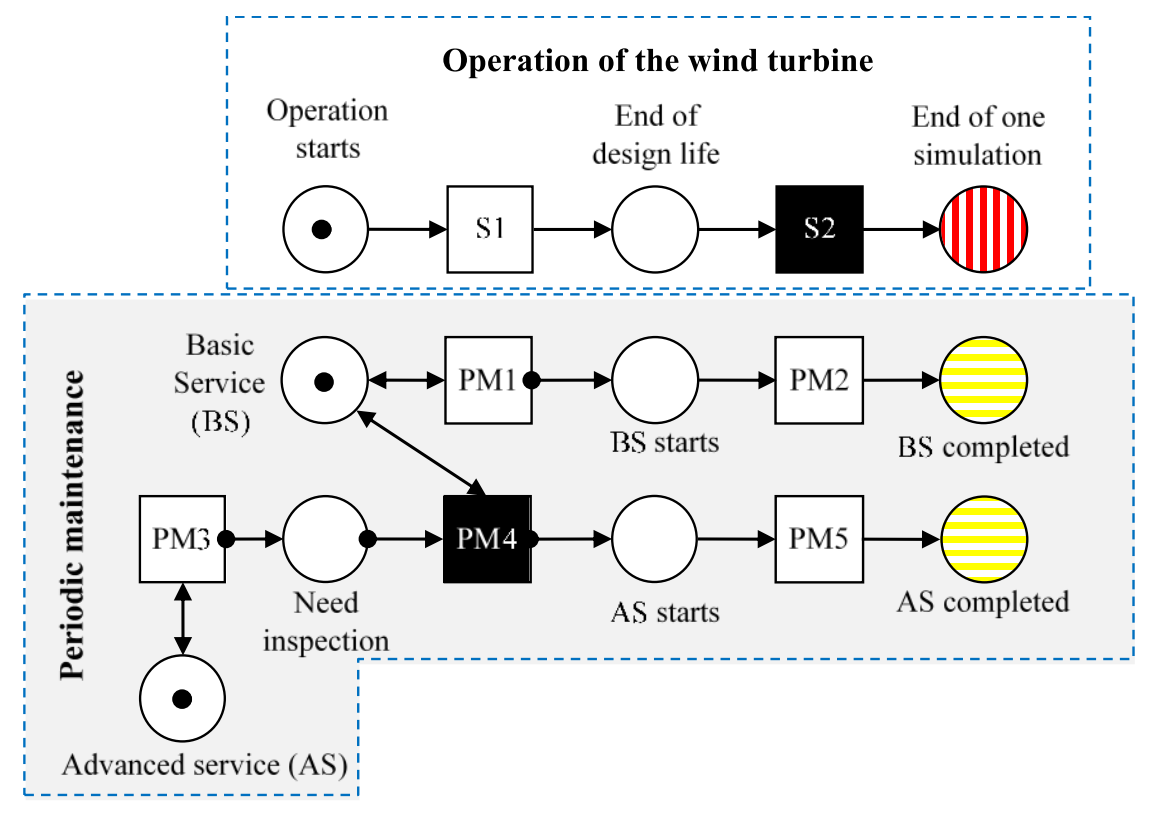

FIGURE 2. Operation Petri net (OPN) (Yan \& Dunnett, 2021). 


\subsection{System Petri Net (SPN)}

In the paper, the SPN is developed to simulate the degradation and the health state of the wind turbine subsystems over time and is modified based on the model developed in (Yan \& Dunnett, 2021) to simulate the occurrence of abnormal conditions that can be detected by the CMS or SCADA system. As mentioned in Section 2, the natural degradation of the turbine's subsystems is indicated by three health states, namely 'Normal operation', 'Subsystem fault', and 'Subsystem failure' as shown in Figure 3. The degradation time from 'normal' to 'fault' is indicated by Transitions ' $\mathrm{W} 1$ ' to 'W6'. Once a token is produced in any of the condition places 'Subsystem fault' except for the rotor, the DPN will be embedded into the model to simulate the fault detection by the CMS as required by the predefined condition. This is because the CMS installed for the OWT is not capable to detect the rotor fault in the study. If the subsystem fault was not detected by the CMS, the fault may continue to develop. Before the failure occurs, new abnormal conditions (e.g. increase in the bearing temperature) which is detectable by the SCADA system could appear. The times taken for them are modelled via Transitions 'W7' to 'W11'. The firing of these transitions will also embed the DPN into the model. Once a subsystem fault is successfully detected by the CMS or SCADA system, the RMPN will be embedded into the model.

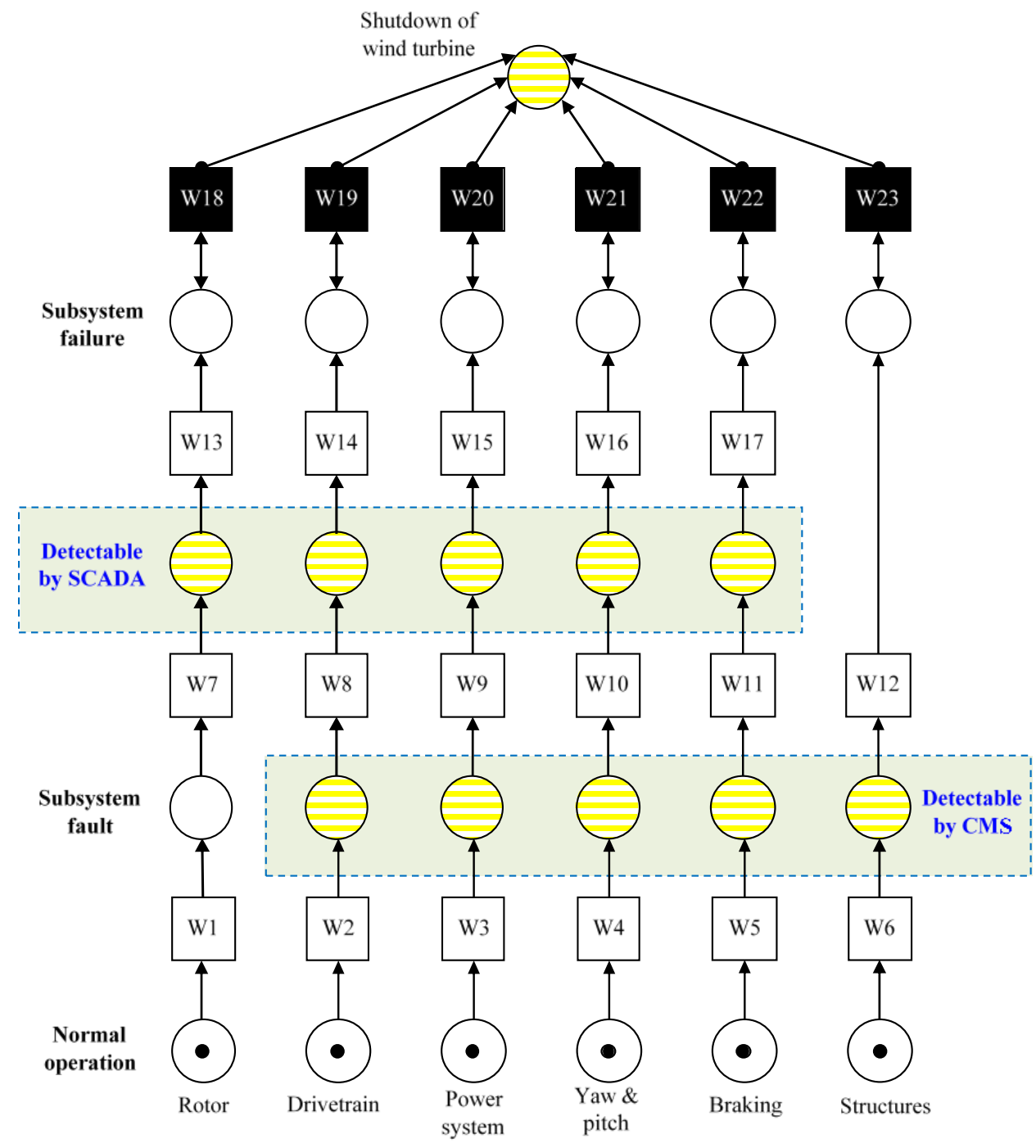

FIGURE 3. System Petri Net (SPN)

If the fault was still not detected or repaired in time, the subsystem will fail eventually as modelled by Transitions 'W12' to 'W17'. In the study, it is assumed that times associated with Transitions 'W1' to 'W17' satisfy Weibull distributions characterised by the parameters listed in Table 3 . The shape parameters $\beta$ in the distributions are assumed to be larger than 1 for all six subsystems in order to describe the increasing 
deterioration of the subsystems over time. Once a token is produced in the place, 'Shutdown of wind turbine', it means a critical subsystem of the turbine has failed. Hence, the RMPN will be embedded into the model.

\begin{tabular}{c|c|c|c|c|c}
\hline \multirow{2}{*}{ Transition } & \multicolumn{2}{|c|}{ Subsystem fault } & \multirow{2}{*}{ Transition } & \multicolumn{2}{c}{ Subsystem failure } \\
\cline { 2 - 3 } \cline { 6 - 6 } & & year & & & year \\
\hline W1 & $\beta=1.2$ & $\eta=8.06$ & W10 & $\beta=1.2$ & $\eta=0.65$ \\
\hline W2 & $\beta=1.2$ & $\eta=10.00$ & W11 & $\beta=1.2$ & $\eta=1.25$ \\
\hline W3 & $\beta=1.2$ & $\eta=4.90$ & W12 & $\beta=1.2$ & $\eta=3.80$ \\
\hline W4 & $\beta=1.2$ & $\eta=4.56$ & W13 & $\beta=1.2$ & $\eta=1.15$ \\
\hline W5 & $\beta=1.2$ & $\eta=8.76$ & W14 & $\beta=1.5$ & $\eta=0.71$ \\
\hline W6 & $\beta=1.2$ & $\eta=8.86$ & W15 & $\beta=1.2$ & $\eta=0.35$ \\
\hline W7 & $\beta=1.2$ & $\eta=2.30$ & W16 & $\beta=1.2$ & $\eta=1.30$ \\
\hline W8 & $\beta=1.5$ & $\eta=2.37$ & W17 & $\beta=1.2$ & $\eta=2.50$ \\
\hline W9 & $\beta=1.2$ & $\eta=1.75$ & - & - & - \\
\hline
\end{tabular}

TABLE 3. Weibull distribution parameters for Transitions 'W1' to 'W17'.

\subsection{Detection Petri Net (DPN)}

In the DPN, as shown in Figure 4, there is only one transition, 'D1', which governs the whole detection process in the net. The arcs, represented by the dashed arrow lines, connect Transition 'D1' with the condition places 'Fault is detected' and 'Fault is not detected'. The probabilities that a token transfers to either of the two places are dependent on the fault detection capability of the CMS or the SCADA system as listed in Table 2. It is worth mentioning that this fault detection capability may be affected by many factors, such as the condition monitoring algorithm, types of the sensors, etc (Yang \& Jiang, 2011). Once a token is produced in the place, 'Fault is detected', the RMPN will then be embedded into the model to prepare the essential maintenance resources and then fix the fault. If the fault is not detected, a token will be produced in Place 'Fault is not detected' and no further action will be taken.

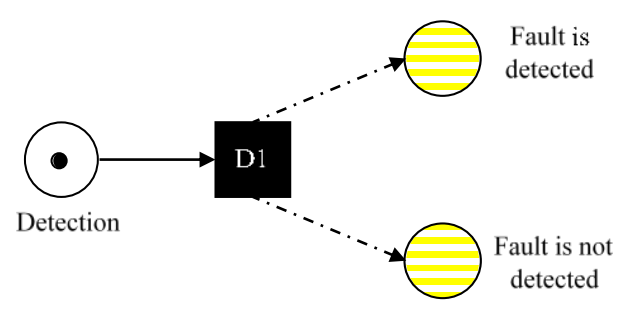

FIGURE 4. Detection Petri Net (DPN) (Yan \& Dunnett, 2021).

\subsection{Recovery and Maintenance Petri Net (RMPN)}

The RMPN shown in Figure 5 is developed in (Yan \& Dunnett, 2021) to simulate the preparation and implementation of the maintenance when any subsystem fails or a subsystem fault is detected by the monitoring systems or revealed during an 'advanced service'. In the figure, once a subsystem failure occurs or a subsystem fault is detected, a repair request will be made via the instant transition, 'M1'. Transition 'M2', which is assumed to be 12 hours, represents the time required to arrange the meeting for planning the maintenance. Also, Transition 'M3' is assumed to be 12 hours, which models the time required for planning and approving the maintenance. After the firing of Transition 'M3', the tokens produced in Places 'Charter vessel' and 'Organise crews, tools and spare parts' will enables the Transitions 'M4' and 'M5', respectively. These two transitions represent the actions for chartering the appropriate maintenance vessel and organising maintenance crews, collecting maintenance tools, and preparing spare parts. Their times are assumed to be 20 days and 10 days respectively. Once all the preparation works are completed, a token will be generated in Place 'Travel to site', which indicates the maintenance vessel is ready to depart. The time for reaching the 
OWT is assumed to be 3 hours which is represented by Transition 'M7'. The onsite preparation time assumed to be 2 hours is denoted by Transition 'M8'. The actual maintenance time, indicated by Transition 'M9', is assumed to be 1 day for repairing all faults or 7 days for recovering the subsystem from the failure. It is worth noting that all parameters used in the models developed above are only for facilitating model development. Theses parameters can be updated according to the actual situations of the OWT in practical applications. Finally, a token will be produced in the place, 'Maintenance completed', and the RMPN will be removed from the model when the maintenance is completed. The new health state of the corresponding subsystem will be fed back to the SPN. This is achieved by removing the token in the corresponding place, 'Subsystem failure' or 'Subsystem fault', and placing a new token in the corresponding place, 'Normal operation', in the SPN.

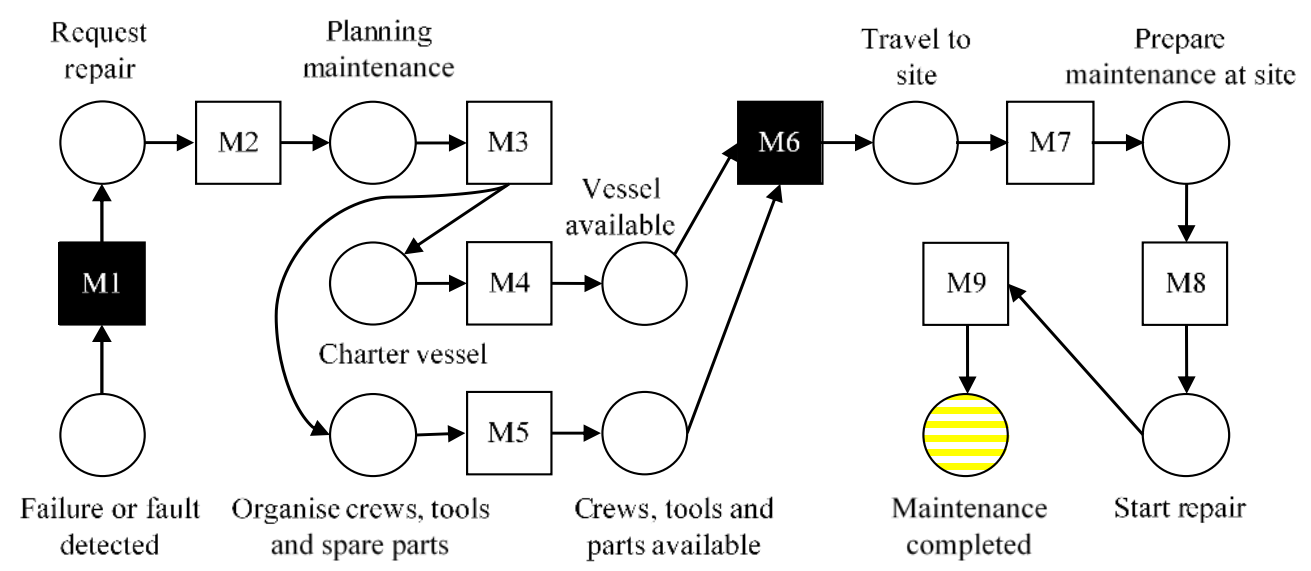

FIGURE 5. Recovery and Maintenance Petri Net (RMPN) (Yan \& Dunnett, 2021).

\section{Simulation results and discussions}

In order to evaluate the effectiveness of the monitoring systems on the wind turbine, the PN models proposed in the previous section can be used for simulation. The following are the simulation assumptions:

- $\quad$ The CMS and the SCADA system have no hardware reliability issues.

- $\quad$ The subsystem fault and failure caused by natural disasters are not considered.

- The impact of the weather condition on the maintenance is not considered.

- $\quad$ The health state of a subsystem after maintenance is regarded as good as new.

- $\quad$ The false alarms generated by monitoring systems are not considered.

- $\quad$ The 'Advanced service' and 'Basic service' are assumed to be conducted every 2 years and 6 months respectively.

Then, based on the data listed in Tables 1, 2, and 3, the simulation calculation is carried out. It is found that the simulation results finally converge to stable values after about 50,000 simulations. To ensure the reliability and convergence of the calculation results, 100,000 simulations will be performed for all the simulations in the research.

The effectiveness of the CMS and the SCADA system installed on the wind turbine is investigated. The average number of failures and the number of faults detected by each monitoring system and the average times of maintenance conducted within the design life of the wind turbine are calculated via the simulation. The calculation results are listed in Table 4. 


\begin{tabular}{c|c|c|c|c|c}
\hline Subsystems & $\begin{array}{c}\text { Failure } \\
\text { recovered }\end{array}$ & $\begin{array}{c}\text { Fault } \\
\text { repaired after } \\
\text { being } \\
\text { detected }\end{array}$ & $\begin{array}{c}\text { Fault repaired } \\
\text { after full } \\
\text { inspection }\end{array}$ & $\begin{array}{c}\text { Fault } \\
\text { detected by } \\
\text { CMS }\end{array}$ & $\begin{array}{c}\text { Fault } \\
\text { detected by } \\
\text { SCADA }\end{array}$ \\
\hline Rotor & 0.142 & 0.357 & 1.565 & 0.000 & 0.363 \\
Drivetrain & 0.001 & 1.801 & 0.135 & 1.766 & 0.047 \\
Power system & 0.009 & 3.824 & 0.241 & 3.696 & 0.156 \\
Bearing system & 0.007 & 4.246 & 0.148 & 3.964 & 0.311 \\
Braking system & 0.009 & 2.079 & 0.140 & 2.026 & 0.066 \\
Structures & 0.044 & 1.990 & 0.163 & 2.006 & 0.000 \\
\hline Sum & 0.212 & 14.297 & 2.392 & 13.458 & 0.945 \\
\hline
\end{tabular}

TABLE 4. The average number of subsystem failures and subsystem faults

From the table, it is found that the number of subsystem failures causing the shutdown of the turbine within its lifetime is 0.212 although 16.689 subsystem faults are repaired in total before developing to failure. Also, from the simulation results obtained, the effectiveness of the monitoring systems defined as the probability of the fault being detected $(P d)$ can be calculated via equation (5.1).

$$
P_{d}=\frac{\text { Fault detected by } C M S+\text { Fault detected by } S C A D A}{\text { Failure repair }+ \text { Total fault repaired }}
$$

It is found that the effectiveness of the monitoring system consisting of the CMS and the SCADA system is about 0.852 . In addition, $93.44 \%$ of the faults detected are found by the CMS, which indicates the effectiveness of the SCADA system being used as an additional monitoring system is limited. However, the installation of the CMS on an offshore wind turbine could be expensive (García Márquez et al., 2012; Yang et al., 2014). Hence, the actual effectiveness of the SCADA system is important to evaluate whether the wind turbine only monitored by the SCADA system could be more cost effective than the one installing both the CMS and the SCADA system. In addition, as some of the SCADA systems generate false alarms too frequently, the operators may decide to ignore the alarms generated by the SCADA system (Yang et al., 2014). Therefore, the impact of removing the fault detection ability of the SCADA system on the wind turbine should also be investigated.

The simulations are then conducted for the wind turbine system without the CMS and the one without the SCADA detection respectively. By assuming the average costs for repairing a subsystem failure, a fault detected by monitoring system, and a fault revealed during period maintenance to be $£ 40,000, £ 12,000$, and $£ 5,000$ respectively, the overall cost for maintaining the availability of the wind turbine through its lifetime can be calculated and the results are listed in Table 5. It should be noted that these costs are deduced based on (McMillan \& Ault, 2009; Le \& Andrews, 2016; Leigh \& Dunnett, 2016; Yan \& Dunnett, 2021).

From the results in Table 5, it is found that if only the SCADA detection is adopted, most of the faults will be revealed during the periodic maintenance rather than detected by the monitoring system. Also, the number of failures is increased by about $280 \%$ without the CMS. This will certainly lead to a longer downtime and a lower availability of the wind turbine. On the other hand, it is noticed that the combined use of the CMS and the SCADA system could save about $£ 160,000$. It also suggests that if the cost of CMS is over $£ 160,000$, it will not be cost-effective based on the data used in the study if we do not consider about the availability of the wind turbine. 


\begin{tabular}{|c|c|c|c|c|}
\hline $\begin{array}{l}\text { Monitoring system } \\
\text { configuration }\end{array}$ & $\begin{array}{l}\text { Failure } \\
\text { repaired }\end{array}$ & $\begin{array}{l}\text { Fault repaired } \\
\text { after being } \\
\text { detected }\end{array}$ & $\begin{array}{l}\text { Fault repaired } \\
\text { after full } \\
\text { inspection }\end{array}$ & $\begin{array}{l}\text { Overall } \\
\text { cost }(£)\end{array}$ \\
\hline CMS and SCADA detection & 0.212 & 14.297 & 2.392 & $268,351.21$ \\
\hline SCADA detection only & 0.798 & 5.458 & 8.708 & $428,146.45$ \\
\hline CMS only & 0.592 & 13.338 & 2.839 & $410,870.13$ \\
\hline
\end{tabular}

TABLE 5. The overall maintenance cost with different monitoring system configurations

On the other hand, if the operators decide to completely ignore the alarms from the SCADA detection and only rely on the detection of the CMS, the overall maintenance cost will be increased by about $£ 140,000$, which is similar to the cost when only the SCADA detection is adopted. Therefore, the effective of SCADA should not be neglected even if the CMS is installed on the wind turbine. However, a better monitoring algorithm might be necessary to reduce the false alarms generated by the SCADA system.

\section{Conclusions}

To achieve an optimal design of a wind turbine condition monitoring system, a new PN model is developed in this paper. With the aid of the model developed, the effectiveness of the CMS and the SCADA system ensuring the reliability and availability of the offshore wind turbine and their impact on the maintenance cost are studied and assessed. From the research reported above, the following conclusions can be made:

1. Using the CMS on the offshore wind power is an effective way to reduce the maintenance cost and improve the availability of the wind turbine. However, the associated cost of the CMS should not be higher than $£ 160,000$ based on the data used in the research.

2. Periodic maintenance is essential to achieve the economical operation of OWTs especially when there is no CMS installed.

3. The fault detection by the SCADA system should not be ignored for achieving an optimal wind turbine condition monitoring system.

It should be noted that the failure of the monitoring systems, the possible false alarms they could generate and the responses to them are not modelled in the study. In the future, their impact on the O\&M of the OWTs will be investigated. In addition, the impact of extreme environmental events such as earthquakes and typhoons, on the reliability and availability of OWTs should also be considered.

\section{References}

ABDOLLAHZADEH, H., ATASHGAR, K., \& ABBASI, M. 2016. Multi-objective opportunistic maintenance optimization of a wind farm considering limited number of maintenance groups. Renew. Energy, 88, 247-261.

BBC 2021. Climate change: EU to cut CO2 emissions by 55\% by 2030 - BBC News. BBC NEWS. URL https://www.bbc.co.uk/news/world-europe-56828383.

DALGIC, Y., LAZAKIS, I., DINWOODIE, I., MCMILLAN, D., \& REVIE, M. 2015. Advanced logistics planning for offshore wind farm operation and maintenance activities. Ocean Eng., 101, 211-226.

GARCÍA MÁRQUEZ, F.P., TOBIAS, A.M., PINAR PÉREZ, J.M., \& PAPAELIAS, M. 2012. Condition monitoring of wind turbines: Techniques and methods. Renew. Energy, 46, 169-178.

GWEC 2019. Global wind energy council report 2018. Wind Glob. Counc. Energy, 1-61.

GWEC 2021. Global Wind Report | Gwec. Glob. Wind Energy Counc., 75. 
KUSEYRI, İ.S. 2015. Condition monitoring of wind turbines: Challenges and opportunities Condition monitoring of wind turbines : Challenges and opportunities. ISITES2015. Valencia - Spain, pp. 116- 126.

LE, B. \& ANDREWS, J. 2016. Modelling wind turbine degradation and maintenance. Wind Energy, 19, $571-591$.

LEIGH, J.M. \& DUNNETT, S.J. 2016. Use of Petri Nets to Model the Maintenance of Wind Turbines. Qual.Reliab. Eng. Int., 32, 167-180.

MAY, A., MCMILLAN, D., \& THÖNS, S. 2015. Economic analysis of condition monitoring systems for offshore wind turbine sub-systems. IET Renew. Power Gener., 9, 900-907.

MCMILLAN, D. \& AULT, G.W. 2009. Specification of reliability benchmarks for offshore wind farms. Safety, Reliab. Risk Anal. Theory, Methods Appl. - Proc. Jt. ESREL SRA-Europe Conf., 4, 2601- 2606.

NAKAGAWA, T. 2005. Maintenance Theory of Reliability. , Maintenance Theory of Reliability, Springer Series in Reliability Engineering. London: Springer-Verlag.

SANTOS, F., TEIXEIRA, Â.P., \& SOARES, C.G. 2015. Modelling and simulation of the operation and maintenance of offshore wind turbines. Proc. Inst. Mech. Eng. Part O J. Risk Reliab., 229, 385- 393.

SIEGEL, D., ZHAO, W., LAPIRA, E., ABUALI, M., \& LEE, J. 2014. A comparative study on vibrationbased condition monitoring algorithms for wind turbine drive trains. Wind Energy, 17, 695-714.

TCHAKOUA, P., WAMKeUE, R., OUHROUCHE, M., SlaOUI-HASNAOUI, F., TAMEGHE, T., \& EKEMB, G. 2014. Wind Turbine Condition Monitoring: State-of-the-Art Review, New Trends, and Future Challenges. Energies, 7, 2595-2630.

YAN, R. \& DUNNETT, S. 2021. Improving the Strategy of Maintaining Offshore Wind Turbines through Petri Net Modelling. Appl. Sci., 11, 574.

YANG, W. \& JIANG, J. 2011. Wind turbine condition monitoring and reliability analysis by SCADA information. 2011 Second International Conference on Mechanic Automation and Control Engineering. IEEE, pp. 1872-1875.

YANG, W., LANG, Z., \& TIAN, W. 2015. Condition Monitoring and Damage Location of Wind Turbine Blades by Frequency Response Transmissibility Analysis. IEEE Trans. Ind. Electron., 62, 6558- 6564.

YANG, W., TAVNER, P.J., CRABTREE, C.J., FENG, Y., \& QIU, Y. 2014. Wind turbine condition monitoring: technical and commercial challenges. Wind Energy, 17, 673-693. 